\title{
Modern immunosuppressive agents after heart transplantation.
}

\author{
Maravić-Stojković Vera*, Stojković Branislav, Perić Miodrag \\ Dedinje Cardiovascular Institute, School of Medicine, University of Belgrade, Belgrade, Serbia
}

\begin{abstract}
The purpose of this article is to provide insight into current trends of immunosuppression in heart transplantation. Currently available classes of drugs: steroids, antimetabolites, polyclonal and monoclonal antibodies, calcineurin inhibitors, proliferation signal inhibitors, have been described in detail. These drugs are in use either in a prophylactic manner or as a part of the maintenance immunosuppressive regimen. Induction therapy provides immune suppression as a key point to induce graft tolerance. The backbones of immunosuppression in organ transplant recipient: cyclosporine $A$ and tacrolimus, have been widely used as maintenance therapy in combination with adjunctive immunosuppressant. We also mentioned the immunosuppressive agents under development: lymphocyte modulation therapy, tolerance induction drugs, gene therapy possibilities and xenotransplantation as an option to overcome organ shortage. After heart transplantation, immunosuppressive medications are used to reduce the risk of the donor's organ rejection. The focus of medical therapy following a heart transplantation is to prevent graft rejection, since the host's immune system is programmed to attack foreign antigens, which could cause injuries and lifethreatening conditions. The possibility of rejection is everlasting, which inevitably demands the use of immunosuppressive drugs and raises the risk of unwanted side effects. Post-operative complications include hyperacute, acute or chronic rejection, as well as post-transplant lymphoproliferative disorders, and permanent susceptibility to infections. Unfortunately, some of the recipients developed renal failure or malignant diseases due to the aggressive immunosuppression. Immunosuppressant agents differ not only in terms of mechanisms of action, but also in terms of unwanted side effects. This offers an opportunity to combine drugs with synergistic actions and a chance to successfully tailor anti-rejection therapy.
\end{abstract}

Keywords Steroids, Antimetabolites, Antibodies, Calcineurin inhibitors, Proliferation signal inhibitors.

Accepted on August 28, 2017

\section{Introduction}

Transplantation medicine is a life-saving procedure for patients with end stage organ diseases. Since the first successful kidney transplantation was prepared in the $1950 \mathrm{~s}$, and particularly since the introduction of potent and selective immunosuppressive agents in the 1980s, great progress has been made in the graft preservation and patient survival [1]. Heart transplantation (HTx) was at first an excellent experiment on animals, which evolved into a successful therapy for patients with severe heart failure [2-3]. On a global level, approximately 3,500-4,000 heart transplants are performed annually, half of which in the United States. About 800,000 people have been classified in the NYHA functional Class IV, and most of them are in a waiting list for heart transplant [4]. Over the past twenty years, several studies reported about improved survival rates following the orthotopic HTx: 1-year survival of $88.0 \%$ (males), 86.2\% (females), 3year survival of $79.3 \%$ (males), $77.2 \%$ (females) and 5-year survival of $73.2 \%$ (males), $69.8 \%$ (females) [5]. The world's longest living heart transplant recipient has been alive and well after 31 years [6]. This increasing success is largely a result of advances in several areas, including (but not limited to):

Tissue typing and donor-recipient matching,
Careful donor evaluation, organ procurement, organ preservation, and recipient preparation;

Perfect surgical technique;

Use of antimicrobial prophylaxis or preemptive treatment to prevent infection or its sequels;

Individualized immunosuppression that balances prevention and treatment of graft rejection with minimal risk of toxic side effects;

Replacement of the aggressive immunosuppression with low doses of the synergistic drugs to reduce malignancy.

After HTx, the patient takes immunosuppressive medications throughout the entire lifetime of the graft, i.e. the patient. The main goal is to achieve immunological tolerance, i.e. unresponsiveness of the immune host system to the foreign antigens. Unfortunately, such medicaments are not discovered so far, and the next step is to minimize the risk of organ rejection. To accomplish this task, we use genetic investigations, the procedures which allow us to pick out the analogous tissue antigens between donor and recipient. After appropriate matching and starting the induction immunosuppression, close monitoring and frequent dose adjustment is necessary. In order to detect rejection, the patient is permanently monitored, including regular heart muscle 
Citation: Maravic-Stojkovic V, Stojkovic B, Peric M. Modern immunosuppressive agents after heart transplantation. Curr Trend Cardiol. $2017 ; 1(2): 41-48$.

biopsy and some sophisticated blood tests. Usually, biopsy is performed once a week during the first postoperative month (or whenever the rejection is strongly suspected), followed by a more relaxed schedule later on.

The risk of rejection never fully goes away, and the patient needs to receive immunosuppressive agents for the rest of his/her life. This may cause unwanted side effects, such as hyperacute, acute or chronic rejection, as well as posttransplant lymphoproliferative disorders (PTLD), or permanent susceptibility to all kinds of infections. Unfortunately, some recipients developed renal failure or malignant diseases due to the over-aggressive immunosuppressive treatment.

\section{Immunosuppressive Drugs}

Immunosuppression has evolved gradually since it was first introduced in the middle of the previous century (Figure 1). Currently available classes: corticosteroids, antimetabolites, poly- and mono-clonal antibodies, calcineurin inhibitors (CNI's) and proliferation signal inhibitors, have been described and presented in Table 1.

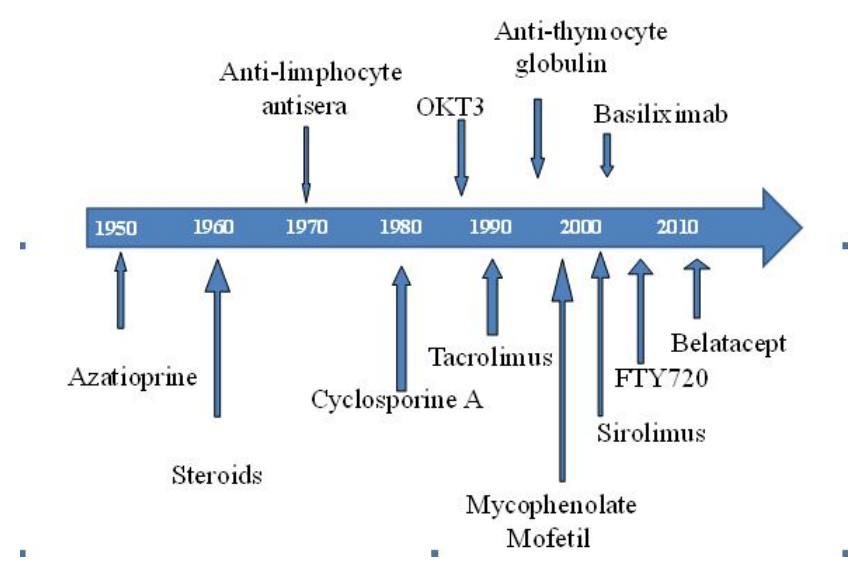

Figure 1. Time line for the introduction of immunosuppression medications. Legend: FTY720 - Fingolimod, OKT3 - monoclonal antibody against CD3 molecule.

These drugs are in use either in a prophylactic manner or as a part of the maintenance immunosuppressive regimen (basic immunosuppressants). There are several concepts of posttransplantation immunosuppressive regimen, which can be used as a guide to this ever-changing issue. Induction therapy provides immunologic ablation with upstream antibody therapy as a prelude to induce graft tolerance. The backbones of immunosuppression in organ transplantation recipient (such as cyclosporine A and tacrolimus) have been widely used as a maintenance therapy, as well, in combination with adjunctive immunosuppressants. Several other immunosuppressive agents are under development: lymphocyte modulation and tolerance induction drugs, as well as gene therapy.

In this article, we provided an overview of immunosuppressant drugs in cardiac transplantation, listing the mayor classes of immunosuppressive agents used for HTx.
Approved immunosuppressive drugs are cited in (Table 1).

Table 1. Mayor Classes of Immunosuppressive Drugs.

\begin{tabular}{|c|c|c|c|}
\hline S.no & & Generic Names & Trade Names \\
\hline 1 & & Corticosteroids & \\
\hline \multirow[t]{4}{*}{2} & & Antimetabolites & \\
\hline & 2.1. & Azatioprin & Imuran \\
\hline & 2.2 . & Mycophenolate mofetil & CellCept \\
\hline & 2.3. & Mycophenolic acid & Myfortic \\
\hline \multirow[t]{3}{*}{3} & & $\begin{array}{l}\text { Polyclonal and Monoclonal } \\
\text { Antibodies }\end{array}$ & \\
\hline & 3.1. & Therapy of acute rejection & ALG, ATG, ATGAM, OKT3 \\
\hline & 3.2. & Immunoprophylaxis & Duclizumab, Simulect \\
\hline \multirow[t]{3}{*}{4} & & Calcineurin Inhibitors & \\
\hline & 4.1. & Cyclosporine A & Neoral \\
\hline & 4.2 & Tacrolimus & FK 506, Prograf, Advagraf \\
\hline \multirow[t]{3}{*}{5} & & Proliferation Signal Inhibitors & \\
\hline & 5.1. & Sirolimus & Rapamune \\
\hline & 5.2. & Everolimus & Certican \\
\hline \multirow[t]{2}{*}{6} & & Lymphocyte Modulation & \\
\hline & 6.1. & FTY720 & Fingolimod \\
\hline 7 & & Tolerance Induction Therapy & \\
\hline 8 & & Gene Therapy & \\
\hline 9 & & Xenotransplantation & \\
\hline
\end{tabular}

\section{Corticosteroids}

Corticosteroids (Ste) were introduced as the basic drug from the very beginning of solid organ transplantation. They are the most utilized non-calcineurin inhibitor immunosuppressive agents after HTx [5]. Steroids are non-specific immunosuppressants. They are known to produce immunosuppression by way of various mechanisms, which results in a potent and generalized anti-inflammatory response. Steroids control the immune response by blocking T-cell and antigen-presenting cell for cytokine expression, especially interleukin (IL)-1, IL-3, IL-4, and IL-5, etc. To prevent acute rejection, steroids are a part of triple therapy, firstly as induction therapy, and after HTx they continue to be used as maintenance therapy [7]. Dosages vary depending on the individual patient's compliance, but the typical protocol includes: a bolus dose of 500-1000 mg of methylprednisolone just prior to the surgery (or before the aortic cross-clamp is released), followed by reduction over the next months to the minimal dose of $25-50 \mathrm{mg} /$ day.

Steroids produce frequent side effects, mainly by suppression of the hypothalamic-pituitary-adrenal (HPA) axis, hyperglycemia, hyperlipidemia, hypertension, osteoporosis, increased risk of gastric ulcers, or risk of fungal and bacterial infections [8]. Due to many side effects, there has been 
significant interest in early steroid withdrawal (as early as 6 months) after solid organ transplantation [9]. There are numerous reports that early steroid withdrawal improves patient's quality of life and decreases the malignancy rate. But some patients are less likely to tolerate steroid withdrawal. Successful weaning from steroids requires a patient subgroup which is immunologically privileged, and the non-occurrence of steroid induced toxicity promotes graft survival. However, in those individuals a close long-term surveillance seems warranted [10].

\section{Antimetabolites}

Across the world, the most frequently used antiproliferative agents after transplantation are: azathioprine (Aza) and mycophenolate mofetil (MMF).

2.1 Azathioprine was the cornerstone of immunosuppressive therapy before the cyclosporine era. The first antimetabolite used in solid organ transplant was azathioprine, but its use has declined over two decades. Azathioprine is an imidasolyl derivative of mercaptopurine which set against purine metabolism. Typical dosage is $1-2 \mathrm{mg} / \mathrm{kg} /$ day. Significant myelosuppression and hepatotoxicity were suggested as side effects, and these are responsible for the decreased clinical use. More recently, MMF replaced Aza as the first-line antiproliferative drug [11], although Aza is several times cheaper than MMF.

2.2 Mycophenolate mofetil (MMF, CellCept) and 2.3 Mycophenolic acid (MPA, Myfortic) interrupt the de novo purine nucleotide synthesis via disruption of the inosine monophosphate dehydrogenase (IMPDH) action. That way, they inhibit DNA replication in $\mathrm{B}$ and $\mathrm{T}$ lymphocytes and blocked alternate salvage pathways. MMF and MPA are potent, selective, non-competitive and reversible inhibitors of enzyme IMPDH. Hence, they inhibit the proliferation of lymphocyte and thus prevent graft rejection, while sparing the salvage pathway. Both formulations selectively suppress lymphocytes.

Treatment starts with 2 to $3 \mathrm{~g}$ daily for MMF, or $720 \mathrm{mg}$ for MPA, spliced in 2 doses. In cardiac transplantation, there are some limitations in mycophenolate mofetil usage, since gastrointestinal discomfort and hematological disorders limit the application in initial immunosuppression (platelet count $<75 \times 109 / \mathrm{L}$, diarrhea). MMF is an immediately released product, as opposed to MPA, which has a postponed release. Enteric coated formulation of MPA delays the moment of releasing the mycophenolic acid in jejunum and small bowel, which increases the Myfortic exploitation.

The introduction of MMF in treating patients with solid organ transplantation has shown significant reduction in 1-year mortality and reduced rates of rejection. Furthermore, MMF is also associated with improvements in the intracoronary luminal area as assessed by intravascular ultrasound, which suggests that it may provide long-term benefits in reducing cardiac allograft vasculopathy (CAV) [12-13].

\section{Polyclonal and monoclonal antibodies}

The purpose of antibody therapy is to postpone the introduction of maintenance therapy or to prepare conditions for the elimination of an immunosuppressive drug, particularly corticosteroids. Antibody therapy can be considered as a receptor-depleting therapy or modulatory therapy (or both). Major products have been listed in (Table 2).

Table 2. Polyclonal and Monoclonal Antibodies.

\begin{tabular}{|c|c|c|c|}
\hline S no & & Generic Names & Trade Names \\
\hline \multirow[t]{4}{*}{3.1} & & Polyclonal antibodies & \\
\hline & 3.1 .1 & Antilymphocite Globulin & ALG, ATGAM \\
\hline & 3.1 .2 & Antithymocyte Globulin & ATG \\
\hline & 3.1 .3 & Muromonab-CD3 & Orthoclone OKT3 \\
\hline \multirow[t]{6}{*}{3.2} & & Monoclonal antibodies & \\
\hline & 3.2 .1 & Duclizumab & Zenapax \\
\hline & 3.2 .2 & Basiliximab & Simulect \\
\hline & 3.2 .3 & Campath-1H & Alemtuzumab \\
\hline & 3.2 .4 & Rituximab & Mabtera \\
\hline & 3.2 .5 & CTLA-4-Ig & Belatacept \\
\hline
\end{tabular}

3.1.1 Polyclonal products mentioned previously under the name Antilymphocyte Globulin (ALG), and Antithymocyte Globulin (ATGAM, of equine origin) are not longer commercially available, although ATGAM could be ordered upon special request.

3.1.2 Antithymocyte Globulin (ATG) is a polyclonal product with antibodies against several epitopes on the human $\mathrm{T}$ lymphocytes (CD2, CD3, CD4, CD8, CD28, CD45 and T-cell receptor), as well as against CD16 located on monocytes and natural killer cells (NK cells) cultivated on a rabbit as a host [14]. The mechanism of action is different, since these antibodies cause internalization of the cell surface receptors, Tcell apoptosis and antibody mediated cytolysis.

After administration of the depleting globulins, the long-lasting effects are profound and could be present several weeks after initialization of antibody therapy. ATG use can induce "cytokine release syndrome" which means high body temperature (over $39^{\circ} \mathrm{C}$ ), chills and possibly rigors, tachypnea, tachyarrhythmia, bronchospasm and gastrointestinal discomfort. Side effect can include a "first-dose" effect, which is related to the numerous cytokines released from lymphocytes, monocytes and NK cells. Dosage ranges from 1.5 to $5.0 \mathrm{mg} / \mathrm{kg}$ in a single infusion over 4 to 6 hours for $3-5$ days.

3.2.1. Muromonab-CD3 (OKT3) is an immunosuppressant approved to reduce acute organ rejection, especially in the case of a glucocorticosteroid-resistant rejection after solid organ grafting. It is a monoclonal antibody of murine origin raised against the CD3 receptor of $\mathrm{T}$ lymphocytes. It binds to the $\mathrm{T}$ cell receptor and CD3-complex, initially leading to lymphocyte 
Citation: Maravic-Stojkovic V, Stojkovic B, Peric M. Modern immunosuppressive agents after heart transplantation. Curr Trend Cardiol. $2017 ; 1(2): 41-48$.

activation, but subsequently inducing blockage and apoptosis of activated T-cells [15].

OKT3 is appointed for the therapy of acute and steroidresistant rejection of allogeneic renal, heart and liver transplants. Later on, prophylactic use is prohibited following cardiac transplantation since post-transplant lymphoproliferative syndrome was found to occur more frequently in those patients. The cytokine release syndrome is identical as in the treatment with ATG. Dosage is $5 \mathrm{mg}$ /day intravenously for 10-14 days.

3.2.2. Duclizumab (Zenapax) and 3.2.3. Basiliximab (Simulect) are chimeric monoclonal antibodies against $\alpha$-chain (CD25) IL-2 receptor T-cells. As of 2009, Zenapax production was discontinued due to commercial reasons.

Basiliximab is an immunosuppressive agent with high specificity to the $\alpha$-chain of IL-2 receptor on the surface of T cells which act by blocking activated lymphocytes [16]. Simulect has the FDA's approval for the prophylaxis of acute organ rejection in patients with renal transplantation when used as part of a triple immunosuppressive regimen that includes CsA, MMF and corticosteroids. Its use is recommended for other solid organ transplant recipients, as well. Simulect is registered for intravenous administration only, as a bolus or as infusion over 30 minutes. Simulect should be administrated only two times: first dose $20 \mathrm{mg}$ within 2 hours after starting surgery, and second dose 4 days after transplantation. These saturate receptors and prevent $\mathrm{T}$ lymphocytes from replication, and also from activating the B cells, which are responsible for the production of antibodies, which would bind to the transplanted organ and stimulate an immune response against the graft [17].

3.2.4. Alemtuzumab (Campath-1H) is a humanized monoclonal antibody that is bound to surface of the cell protein CD52. CD52 is a glycoprotein existing on lymphocytes, thymocytes, monocytes and NK cells, but not on plasma cells, granulocytes, platelets or erythrocytes [18]. Carbon model of the Campath-1H is displayed in (Figure 2).

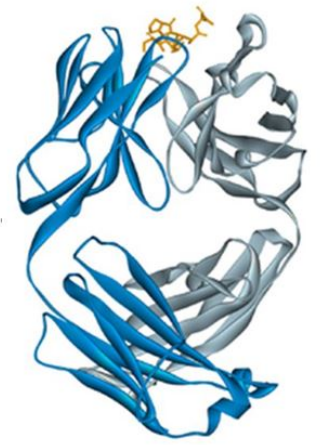

Figure 2: Carbon model of the Campath-1H, humanized monoclonal antibody against CD52 molecule on the surface of mature lymphocytes; Drug is available under trade names: Campath, MabCampath, Campath-1, Lemtrada.

Alemtuzumab is directed against proteins present on the surface of mature lymphocytes, whereas it is not present on the surface of lymphoid stem cells. Alemtuzumab infusion administered in a single dose of $30 \mathrm{mg}$ acts by way of substantial lymphocyte depletion, almost $99 \%$ of CD52bearing lymphocytes are destroyed.

Selective use for induction therapy and preliminary experience in case reports provided us with some promising results in treating refractory rejection after heart transplantation by alemtuzumab. Recently, several investigators reported [19] that use of alemtuzumab as induction therapy in cardiac transplantation permitted a steroid-free maintenance of the immunosuppressive regimen, and substantial reduction dose of tacrolimus and MMF. They administered alemtuzumab prior to cardiac transplantation as induction therapy with no steroids and with low doses of maintenance immunosuppression. Oneyear follow-up have shown that likelihood of rejection was significantly lower for the Campath-1H group $(\mathrm{p}<0.0001)$ [19].

3.2.5. Rituximab (Mabtera) destroys B cells in the body and helps to develop new plasma cells from hematopoietic stem cells. B-cell depleting monoclonal anti-CD20 antibodies act in a selective manner for the treatment of humoral rejection. Carbon model of the Rituximab is available in (Figure 3).

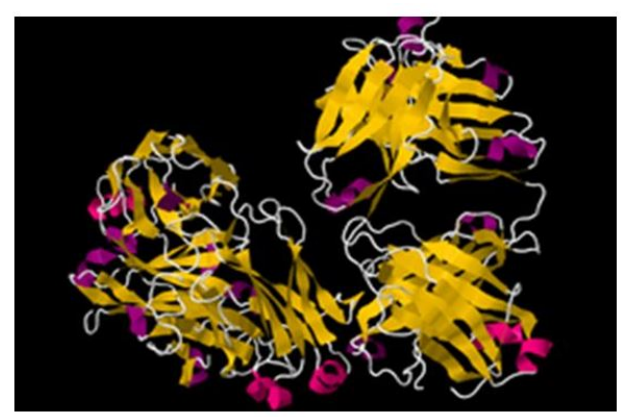

Figure 3: Carbon model of the Rituximab; Chimeric mouse-human monoclonal antibody against CD20 molecule on B-lymphocytes; Drug is available under trade names; Mabtera, Riruxan or Zytux.

Some patients who received a new heart have a high level of antibodies, which could trigger a humoral rejection. Also, early as well as frequent episodes of cellular rejection can trigger the narrowing of the small vessels in the heart, and subsequent vasculopathy. CAV cause irreversible changes on the muscle vessels, which is a life-threatening condition and the reason to select the patient for re-transplantation. Cardiac allograft vasculopathy, PTLD, and malignancy are major factors in reduced long-term survival rates.

The results of an ongoing study conducted in 26 transplantation centers with primary endpoint to explore whether depleting B cells by Rituximab can prevent CAV and damage of the transplanted heart, have produced astonished conclusions. The secondary endpoints included: death, retransplantation or re-listing for transplantation, number of episodes of biopsy proven acute rejection (BPAR), incidence of BPAR, incidence of hyperacute (humoral) rejection, incidence of cellular (acute or chronic) rejection, incidence of any treated rejection. Unexpectedly, investigators have observed a significant increase in percent atheroma volume in patients treated by Rituximab, while survival and treated rejection were 
not different. Be that as it may, the conclusion was that Rituximab should be used with caution in primary unsensitized HTx patients [20].

3.2.6. Belatacept The FDA has recently approved belatacept (CTLA-4-Ig), which is a fusion protein that blocks the CD28 costimulatory pathway [21]. Belatacept is a potent immunosuppressive agent directed against B7 ligands, B7-1 (CD80) and B7-2 (CD86), on the surface of antigen-presenting cells (APC). Those ligands are involved in a signaling pathway known as "signal 2" in the three-signal transplant model of Tcell activation, as it was shown in (Figure 4). It is a selective immunosuppressant, which selectively blocks co-stimulatory molecules present on APC, thereby preventing the activation of naïve T-cells, their proliferation and initiation of cellular rejection in the graft. Since 2011 Belatacept has the FDA's approval for immunosuppressive regimen that include basiliximab, MMF and steroids in Epstein-Barr virus seropositive kidney recipients. Investigations conducted on pancreas and liver transplant recipients are promising. There are evidences that cardiovascular and metabolic side effect are significantly lower, especially blood pressure, level of lipids and de novo developed diabetes. The most important achievement is the fact that Belatacepr could be administrated every few weeks, in contrast to calcineurin inhibitors which should be given twice a day [21]. Furthermore, the use of Belatacept has so far been studied on patients with other types of organ transplantation including heart, lung, and abdominal transplantation.

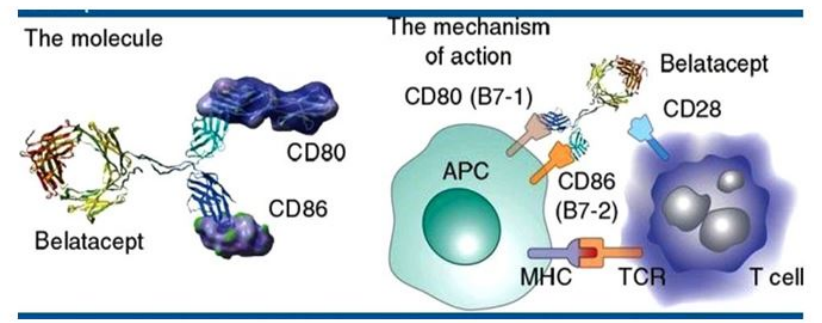

Figure 4: The "signal 2" blocked by Belatacept in graft preservation18. Legend: APC - antigen presenting cell, CD28 costimulatory molecule on T cell, CD80 - costimulatory molecule on APC (also known as B7-1), CD86 - another costimulatory molecule on APC (also known as B7-2), MHC - mayor histocompatibility complex, TCR $-\mathrm{T}$ cell receptor.

\section{Calcineurin inhibitors}

The new class of immunosuppressive agents was introduced during 1970s, the so-called "calcineurin inhibitors" (CNIs). Those drugs form the backbone of immunosuppression (IS) in organ transplantation. Currently, two CNIs are available: cyclosporine A (CsA) and tacrolimus (FK 506, Tac). Calcineurin inhibitors such as cyclosporine and tacrolimus, are routinely used for IS following heart transplantation in conjunction with an antiproliferative agent, with or without maintenance steroids [22]. The advantage of these drugs over cytotoxic immunosuppressants is that their action s specifically directed to the immune system, not affecting other rapidly proliferating cells. In combination with other immunosuppressive drugs, CNIs are widely used for their synergistic mechanism of action. Thus, by lowering the dose of CNIs we achieved fewer side effects, but better efficacy of immunosuppression. Modern immunosuppressive therapy, therapy for the 21 st century, is characterized by "three Ss": specificity, selectivity, and synergism.

4.1. Cyclosporine a (CsA, Neoral) is an immunosuppressive drug widely used after solid organ transplantation to suppress the activity of the patient's immune system. Cyclosporine is a cyclic peptide of fungal origin initially extracted from Tolycopladium inflatum. Binding to an immunophylin, the cytosolic protein cyclophilin on the lymphocytes, CsA makes a complex which blocks calcineurin. That way, it blocks the transcription of the interleukin 2 (IL-2) responsible for the Tcell activation. Calcineurin from cytoplasm prevents the transcription factor, the so-called nuclear factor of activated Tlymphocytes (NF-AT), to move into the nucleus, and to activate genes coding IL-2 and other cytokines necessary for cell proliferation. CsA is a specific immunosuppressant directed against T-lymphocytes.

In cardiac transplantation, initial dose varies from $10-15 \mathrm{mg} /$ $\mathrm{kg}$ per day. Close monitoring of blood levels is necessary on a daily basis, starting within 24 hours of the first administration [22]. Oral doses are three times higher than intravenous formulation, since CsA has approximately $80 \%$ bioavailability. Blood level measured 12 hours after ingestion is known as trough level or $\mathrm{C} 0$ level, since oral formulation should be given twice a day. Adjustment of the dose depends on the trough level. During the first two weeks after heart transplantation, trough level should be 400-450 ng/ml, next two weeks 300-350 $\mathrm{ng} / \mathrm{ml}$ and during weeks $4-25$ around $250-350 \mathrm{ng} / \mathrm{ml}$. Lifelong therapy should be carefully tailored, and the target CsA trough level should be $200 \mathrm{ng} / \mathrm{ml}$. In recent years, some investigators prefer to monitor CsA level two hours after drug ingestion, C2 level. They believe that $\mathrm{C} 2$ level is more informative since it closely correlates with total exposure. Target C2 level of $850-1400 \mathrm{ng} / \mathrm{ml}$ should be reached from 0 to 3 months after cardiac transplantation [23].

4.2. Tacrolimus (FK506 or Fujimycin, Prograf, Advagraf) is another immunosuppressive drug widely used instead of cyclosporine A as a part of the maintenance therapy. It exerts its activation by binding to immunophylin known as a FKbinding protein (FKBP 12). The complex tacrolimus-FKBP 12 prevents the calcineurin to dephosphorilate NF-AT and to activate genes to produce IL-2 and other cytokines. Reduction of IL-2 in T-cells inhibits transcription of IL-3, IL-4, IL-8 and various hemotactic factors. Tacrolimus has a similar mechanism of action via calcineurin, calcium ion and calmodulin, but it is much more potent in equal amount than CsA - 100 times. Initial dose of Tac ranges from 0.01 to 0.15 $\mathrm{mg} / \mathrm{kg} /$ day. Monitoring of the blood concentration is necessary, and trough level would be from 10 to $15 \mathrm{mg} / \mathrm{ml}$, depending on time elapsed from the heart transplantation.

Taken together, the two CNIs immunosuppressive drugs have similar pathway blocking activation of T-lymphocytes and other immunocompetent cells, thus preventing the graft damage, but they differ substantially in toxicity and side effects. It is well known that CsA causes hypertension and 
hyperlipidemia much more frequently that Tac, but Tac appear to cause de novo diabetes mellitus [24], neurologic and kidney disorders. It seems that individual patient's compliance dictates which the better choice is, CsA or Tac, having in mind the adverse effect of drugs and individual patient's profile $[25,26]$.

\section{Proliferation Signal Inhibitors}

Proliferation signal or mammalian target-of-rapamycin inhibitors (PSI/mTOR) include two drugs currently available for clinical use: 5.1 sirolimus (Rapamune) and 5.2 everolimus (Certican). They are very potent agents because of their dual mode of action, immunosuppressive and antiproliferative. Both agents have shown efficacy in diminishing the rate of acute rejection [27].

PSI/mTOR inhibitors work in synergy with CNIs and thus permit the minimization of CNIs without compromising efficacy [28]. This approach is advantageous for the majority of heart transplant recipients and might provide a particular benefit in specific cases, such as for patients with cardiac allograft vasculopathy, malignances, PTLD, and in patients who cannot tolerate other immunosuppressive agents [28]. Both of the PSI/mTOR inhibitors are powerful immunosuppressant, but infections are grave side effects during their usage. Doses vary widely, since the half-life is from 24-36 hours and it depends on the individual patient's compliance. Usually, it is $1 \mathrm{mg}$ given per day in adult population, whereas Certican has a pediatric formulation of drug, s well. The use is limited in patients suffering from severe renal impairment, since proteinuria has been observed in nine out of ten patients. Also, these drugs impair the wound healing process, and its usage is not recommended in the first 28 days after transplantation [27].

\section{Lymphocyte modulation therapy}

FTY720 (FTY, Fingolimod) is a derivative of ISP-1 (myriocin), a fungal metabolite extracted from Chinese herb Isaria sinclairii, as well as a structural analog of sphingosine-1phosphate. FTY720, a novel immunosuppressive agent, acts as a modulator of immune trafficking. It prolongs allograft transplant survival in numerous models by inhibiting lymphocyte emigration from lymphoid organs. The mechanism of action is specific and different to other drugs used in solid organ transplantation. FTY disturbs the homing of Tlymphocytes, especially the CD3+, CD4+, and CD8+ T cell lines and reduces their number in peripheral circulation [28]. At first, the data indicated that FTY affected a number of circulating lymphocytes triggering lymphocyte apoptosis. However, recent investigations have shown that FTY initiates the accelerated homing of lymphocytes to lymph nodes and lymphoid tissue. Migratory response is probably an enhanced response to homing chemokines. FTY720 acts selectively by retaining certain lymphocytes in the lymph nodes, reducing the number of lymphocytes that reach the graft/brain where they can cause inflammatory destruction. As a result, lymphocytes cannot recirculate to graft or/and inflammatory tissue, but they are efficient in the lymphoid compartment (Figure 5) [29].
In December 2009, the data from one of the largest Phase III programs conducted on multiple sclerosis were included in the applications for regulatory approval submitted to the European Medicines Agency (EMEA) and US FDA. Approval is sought for the lower $0.5 \mathrm{mg}$ dose, as the results from the studies indicate that this dose has the most positive benefit-risk profile [28-32].

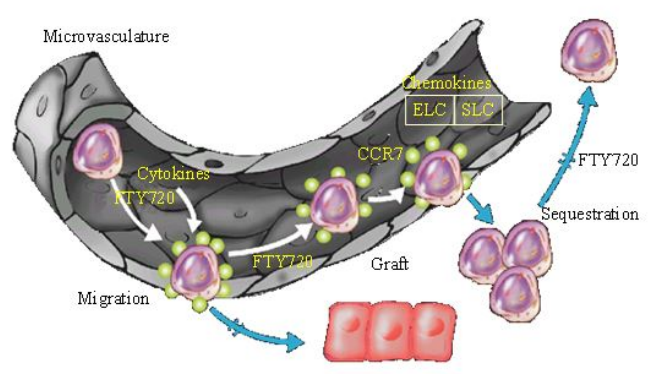

Figure 5: The mechanism of Fingolimod (FTY720) action. Legend: FTY720 - fungal metabolite that retaining certain lymphocytes in the lymph nodes; CCR7: a chemokine receptor required for migration of $\mathrm{T}$ cells; ELC: chemokine necessary for migration and homing of normal lymphocytes; SLC: Secondary Lymphoid-tissue Chemokine.

\section{Summary and Conclusions}

The ideal immunosuppression should be able to prevent or heal rejection and to lower the risk of infection or cancer to its minimum. There are several ways to achieve this goal: to act specifically by depleting lymphocytes, to act selectively by blocking activation of cells, or to redirect the lymphocyte traffic [33]. The successful solid organ transplantation, particularly in the case of heart and liver, was improved with the introduction of potent IS drugs, particularly CNIs inhibitors. As it is shown in Figure 1. the introduction of cyclosporine in the early 1980 s as the backbone of IS regiments, resulted with substantial improvement in the survival of patients with solid organ transplantation. Two decades later other agents were introduced, MMF/MPA with the intention to achieve lowering of the dose of CNIs as maintenance therapy and to prevent early rejection. The biggest movement after 2000 was opportunity to combine several IS drugs as introduction therapy, when alloimune response is most intense. Modern IS agents for induction therapy can be summarized as follows: 1) depleting antibodies (ATG, OKT3, and alentuzumab); or 2) basiliximab or fusion proteins CTLA4-Ig [34]. This can be achieved with or without the use of the induction therapy, which is nowadays used in about one-half of transplant programs. The growing body of evidence rises concerns about the use of OKT3, which is associated with greater risk of lymphoproliferative disorders [15]. The reason for this is the recently acquired evidence of long-term complications linked to certain medications used as induction therapy. This suggests that it is necessary to [3] find the balance between benefit and long-lasting toxicity developed during medical therapy. For this reason, there are suggestions that introducing therapy should be in use selectively only in high sensitized patients. 
The most important is maintenance IS therapy with the objective to produce continuous host-graft tolerance with lowering the risk of malignancies and opportunistic infections. All transplant centers have adopted protocols where triple therapy is in use as a maintenance therapy, starting with corticosteroids, one of the CNIs (cyclosporine or tacrolimus) and an antimetabolite, usually MMF. Corticosteroids are generally used early after Tx until the end of first year by slow tapering of the dose. The withdrawal can be eater fast/early or slow/late. In early withdrawal, the prednisone is discontinued within the first month after HTx. Late withdrawal involves the use of prednisolon for at least 6-12 months and has the advantage of more intense immunosuppression in the first six months when rejection rate is at its highest level $[35,36]$. Minimal use of steroids and early withdrawal are strongly recommended in pediatric transplantation, as these may impair normal growth.

Modern immunosuppression is directed toward the use two or three drugs with distinct mechanisms of action and different side effects. That way, synergistic potential of the IS drugs is combined to achieve strong antirejection effect but to avoid toxicity. Belatacept and FTY720 are very effective, with no additional toxicities, but capable for synergistic action in combination with other drugs. The final goal is to reduce the number of drugs and to achieve safety in monotherapy. In that regard, it is important to have in mind that each patient is a unique person and that tailoring immunosuppressive agents is a lifelong task after solid organ transplantation.

\section{References}

1. Roger VL, Go AS, Lloyd-Jones DM, et al. Heart Disease and Stroke Statistics-2012 Update The American Heart Association. Circulation. 2012;125:2-220.

2. Griepp RB, Ergin MA. The history of experimental heart transplantation. J Heart Transplant. 1984; 3:145.

3. Reiner Körfer. The Heart-Makers: The Future of Transplant Medicine. Germany: LOOKS film and television. 2007.

4. Mehra MR, Canter CE, Hannan MM, et al. The 2016 International society for heart and lung transplantation listing criteria for heart transplantation: A 10-year update. J Heart Lung transplant. 2016; 35:1-23.

5. Botta DM. Heart transplantation-Medscape References. 2014.

6. Heart Transplant Patient OK After 28 Yrs (14 September 2006) CBS News. Retrieved 29 December 2006

7. Niklason LE, Langer R. Prospects for organ and tissue replacement. JAMA. 2001;285:573-6.

8. Danovitch G. Immunosuppressive medications and protocols. In: Danovitch GM, ed. Handbook of Kidney Transplantation. Philadelphia PA: Lippincott Williams\&Wilkins, 2001:82-83.

9. Rosenbaum DH, Adams BC, Mitchell JD, et al. Efects of early steroid withdrawal after heart transplantation. Ann Thor Surg. 2006;82:637-44.

10. Felkel TO, Smith AL, Reichenspurner HC, et al. Survival and incidence of acute rejection in heart recipients undergoing successful withdrawal from steroid therapy. J Heart Lung Transpl. 2002;21:530-9.

11. Becker BN. Mycophenolatemofetil. Transplant Proc. 1999;31:2777-8.

12. Kobashigawa JA, Meiser BM. Review of major clinical trials with mycophenolatemofetil in cardiac transplantation. Transplantation. 2005; 80:S235-43.

13. Passi N, Snead J, Goldstein DJ, et al. Mycophenolate mofetil in Adult Orthotopic Heart Transplant Recipients: How much is To Much? J Heart Lung Transpl. 2016; 35:S422.

14. Meier-Kriesche HU, Li S, Gruessner RW, et al. Immunosuppression: Evolution in practice and trends, 1994-2004. Am J Transplant. 2006; 6:1111-31.

15. Swinnen LJ, Constanyo-Nordin MR, Fisher SG, et al. Increased incidence of lymphoproliferative disorder after immunosuppression with the monoclonal antibody OKT3 in cardiac transplant recipients. $\mathrm{N}$ Engl $\mathrm{J}$ Med. 1990;323:1723-8.

16. Beniaminovitz A, Itescu S, Lietz K, et al. Prevention of rejection in cardiac transplantation byblockade of the interleukin-2 receptor with a monoclonal antibody. N Engl J Med. 2000; 342:613-9.

17. Waldmann TA. Immunotherapy: past, present and future. Nat Med. 2003;9:269-77.

18. Hale G, Xia MQ, Tighe HP, et al. The CAMPATH-1 antigen (CDw52). Tissue Antigens. 1990; 35:118-27.

19. Teuteberg JJ, Shullo MA, Zomak R, et al. Alentuzumab Induction Prior to Cardiac Transplantation with Lower Intensity MaintenanceImmunosuppression: One-year Outcomes. Am J Transplant. 2010;10:382-8.

20. Chandraker A, Kobashigawa J, Stenlik J, et al. Rituximab induction in cardiac transplantation is associated with accelerated coronary artery vasculopathy: CTOT11. Am J Transplant. 2016;16:3-5.

21. Wojciechowski D, Vincenti F. Belatacept in kidney transplantation. Curr Opin Organ Transplant. 2012; 17:640-7.

22. Miller LW. Cardiovascular toxicities of immunosuppressive agents. Am J Transplant. 2002;2:807-18.

23. Taylor DO. Immunosuppressive therapies after heart transplantation: best, better and beyond. Curr Opin Cardiol. 2000;15:108-14.

24. Keogh A. Calcineurin inhibitors in heart transplantation. J Heart Lung Transplant. 2004;23:S202-6.

25. Baran DA, Segura L, Kushwaha S, et al. Tacrolimus monotherapy in adult cardiac transplant recipients: intermediate-term results. J Heart Lung Transplant. 2001;20:59-70.

26. Keogh A. Calcineurin inhibitors in heart transplantation. J Heart Lung Transplant. 2004;23:S202-6.

27. Sehgal SN. Sirolimus: Its discovery, biological properties, and mechanism of action. Transpl Proc. 2003;35:7S-14S.

28. Shapiro R, Young JB, Milford EL. Immunosuppression: Evolution in practice and trends, 1993-2003. Am J Transplant. 2005;59:253-81. 
Citation: Maravic-Stojkovic V, Stojkovic B, Peric M. Modern immunosuppressive agents after heart transplantation. Curr Trend Cardiol. $2017 ; 1(2): 41-48$.

29. Mulgaonkar S, Tedesco H, Oppenheimer F, et al. FTY720/ Cyclosporine Regimens in De Novo Renal Transplanta tion: A 1-Year Dose-Finding Study. American J Transplantation. 2006.

30. Chiba K, Hoshino Y, Suzuki C, et al. FTY720, a novel immunosppressant possessing unique mechanisms. I: Prolongation of skin allograft survival and synergistic effect in combination with cyclosporine in rats. Transplant Proc. 1996;28: 1056-9.

31. Suzuki S, Enosawa S, Kakefunda T, et al. A novel immunosuppressant, FTY720, with a unique mechanism of action, induces long-term graft acceptance in rat and dog allotransplantation. Transplantation. 1996;61:200-5.

32. Nikolova Z, Hof A, Rudin M, et al. Prevention of graft vessel disease by combined FTY720/cyclosporine A treatment in a rat carotid artery transplantation model. Transplantation. 2000;69: 2525-30.

33. Michaels PJ, Espejo ML, Kobashigawa JA, et al. Humoral rejection in cardiac transplantation: risk factors, hemodynamic consequences and relationship to transplant coronary artery disease. J Heart Lung Transplant. 2003;22:58-69.

34. Baran DA. Induction therapy in cardiac transplantation: when and why? Heart Fail Clin. 2007;3:31-41.

35. Miller SN, Finley MP, Li s. Immunosuppression: Evolution in practice and trends, 1993-2003. Ann Arbor. 2004.

36. Kobashigawa JA. Strategies in immunosuppression after heart transplantation. Circulation. 2011;4:111-3.

\section{*Correspondence to}

Maravić-Stojković Vera

School of Medicine Belgrade University

Belgrade, Serbia

E-mailid: vmaravic@ikvbd.com 